\title{
Aceptabilidad Sensorial y Calidad Microbiológica de Bebidas a Base de Arroz y Plasma Bovino y Porcino
}

\author{
Diego F. Tirado, Piedad M. Montero* y Diofanor Acevedo \\ Universidad de Cartagena, Facultad de Ingeniería, Programa de ingeniería de Alimentos, Grupo de \\ Investigación Nutrición, Salud y Calidad Alimentaria (NUSCA), Avenida el Consulado, Calle 30 No. 48-152. \\ Cartagena, Bolívar-Colombia (e-mail: pmargaritamontero@hotmail.com) \\ * autor a quien debe ser dirigida la correspondencia
}

Recibido Feb. 25, 2015; Aceptado May. 15, 2015; Versión final Jun. 28, 2015, Publicado Dic. 2015

\begin{abstract}
Resumen
Se evaluó la aceptabilidad sensorial y calidad microbiológica de bebidas formuladas a base de arroz y fortificadas con proteína proveniente de plasma bovino y porcino. El plasma se extrajo de la sangre por centrifugación y se analizaron las variables de respuesta en función de seis formulaciones con los tipos de plasma adicionado y niveles de fortificación del $14.5 \%, 18.5 \%$ y $29.0 \%$ de plasma. El tipo de plasma adicionado y los niveles de fortificación de las formulaciones afectan significativamente la aceptabilidad sensorial de las bebidas. La bebida fortificada con $29 \%$ de plasma bovino fue el tratamiento con mayor aceptabilidad sensorial. La fortificación de la bebida refrescante a base de arroz con plasma de bovino permitió crear un producto que cumple con un adecuado suministro de aminoácidos esenciales, lo cual evidencia el carácter sinérgico de la combinación de las materias primas usadas en este estudio.
\end{abstract}

Palabras clave: plasma sanguíneo, aceptabilidad sensorial, calidad microbiológica, calidad proteica, aminoácidos

\section{Sensory Acceptability and Microbiological Quality of Rice Based Drinks and Bovine and Porcine Plasma}

\begin{abstract}
Acceptability sensory and microbiological quality of drinks made with rice and fortified with protein from bovine and porcine plasma was evaluated. The plasma was extracted from the blood by centrifugation and the response variables according to six formulations with added plasma types and levels of fortification of $14.5 \%$, $18 ., 5 \%$ and $29.0 \%$ of plasma were evaluated. The type of plasma added and the fortification levels of the formulations significantly affect the sensory acceptability of the beverage. The fortified beverage with $29 \%$ bovine plasma treatment was the one with higher sensory acceptability. Fortification of the refreshing drink made of rice with bovine plasma allowed to formulate a product that gives adequate supply of essential amino acids, which demonstrates the synergistic combination of the raw materials employed in the study.
\end{abstract}

Keywords: blood plasma, sensory acceptability, microbiological quality, protein quality, aminoacids 


\section{INTRODUCCIÓN}

Todos los seres vivos, incluyendo el hombre, deben tener una fuente adecuada de proteínas en su alimentación para crecer y conservarse de manera autónoma. Sin embargo, en muchas partes del mundo, especialmente en los países en vías de desarrollo, resulta poco accesible las fuentes de proteínas debido a su alto costo, en especial las de origen animal, las cuales son consideradas proteínas de buena calidad, debido a la cantidad de aminoácidos esenciales que poseen, por lo que la mayor parte de la población no recibe las raciones necesarias de este nutriente, originando una desnutrición por déficit proteico (Benítez et al., 2002).

En Colombia, como pasa en los demás países de Latinoamérica, la deficiencia de proteína, el hambre y la desnutrición no se presentan por falta de disponibilidad de alimentos, sino por elevados niveles de pobreza, por la baja capacidad de compra de proteína animal y por un régimen dietario poco balanceado, donde los cereales constituyen una parte importante de la dieta que en muchos casos se convierten en la base de la alimentación (Barboza et al., 1994). Una alternativa que intenta mejorar este estado nutricional es la utilización de cereales de consumo masivo como el arroz, que hacen parte de la dieta cotidiana de nuestra población en la elaboración de alimentos fortificados con proteína. Aunque el arroz, tanto pulido como integral, representa un importante aporte de energía, posee un perfil aminoacídico incompleto, siendo la lisina el aminoácido limitante; por esto, se ha recomendado su enriquecimiento con aminoácidos o fuentes de proteína animal con el objeto de poder compensar su deficiencia en aminoácidos esenciales (González et al., 2007).

Lo anterior ha sido incentivo para la búsqueda de fuentes alternas, capaces de ofrecer alimentos altamente proteicos con cualidades organolépticas aceptables, de allí que las investigaciones apunten hacia el desarrollo de nuevos productos no convencionales para ser utilizados en la alimentación humana (Benítez et al., 2002). La sangre de algunos animales como bovinos y porcinos es aprovechada en diversos países para la alimentación humana, incorporándolas en los alimentos como fuente de proteína de bajo costo; ya que además de aportar proteína, poseen propiedades funcionales de aplicación en la industria de alimentos (Camacho et al., 2014). En algunos estudios Julio et al., (2015) evaluaron la calidad y aceptabilidad sensorial de productos cárnicos tipo chorizos formulados con plasma sanguíneo bovino, observando que es posible reemplazar porcentajes de agua por plasma sanguíneo en la formulación de chorizos, para mejorar el rendimiento y aumentar el contenido proteico, sin que ello tenga un efecto negativo en la aceptabilidad sensorial. De esta forma gran cantidad de autores señalan la importancia de utilizar plasma sanguíneo que ayuden a mejorar la calidad nutricional de los alimentos para consumo humano (Julio et al., 2015; Isaza et al., 2010; Benítez et al., 2011; Rodríguez et al., 2011; Figueroa et al., 2012; Gómez et al., 2013; Camacho et al., 2014; Montero et al., 2015). Por todas estas razones el plasma sanguíneo de bovino es actualmente aprovechado en diversos países para la alimentación humana, incorporándolo en los alimentos como fuente de proteína de bajo costo (Parés et al., 2011; Hurtado et al., 2011; Isaza et al., 2010). Estas proteínas plásmicas presentan características favorables para su utilización en la industria de los alimentos, dentro de estas encontramos su alto valor nutritivo, su participación como agente emulsificante, espumante y ligante y su capacidad de formar geles y aumentar la rentabilidad (Camacho et al., 2014).

Desde el punto de vista de su valor nutritivo, la sangre ha sido comparada con las carnes, la leche y los huevos. La sangre posee un $18 \%$ de proteína y tiene los aminoácidos esenciales que requiere el hombre para su normal funcionamiento; sin embargo, de las fracciones de la sangre, el plasma es el producto comercial más utilizado para la formulación de alimentos, puesto que su sabor y color es fácilmente enmascarado (Rangel et al., 1995). El plasma es un líquido translucido más denso que el agua y ligeramente alcalino, con pH de 7,4; en algunos casos puede presentar un ligero color rosado, está constituido por un $90 \%$ de agua y contiene alrededor de $7,2 \%$ de proteína (Bracho et al., 2001). Su singular envergadura en el contenido de proteínas y aminoácidos esenciales hace del plasma sanguíneo de bovino y porcino ingredientes alimenticios de suma importancia, teniendo en cuenta la cantidad de bovinos y porcinos que se sacrifican anualmente y los altos niveles de deficiencia de proteína en el mundo (Julio et al., 2015).

La proteína sanguínea, además de significar una fuente importante de nutrientes con calidad microbiológica debido a la aplicación de adecuados métodos de obtención y tratamientos térmico, tiene la ventaja de reducir los problemas de contaminación ambiental asociados con la descarga de la sangre al medio ambiente por parte de las plantas de beneficio (Julio et al., 2015; Benítez et al., 2011; Benítez et al., 2002; Bracho et al., 2001). Teniendo en cuenta lo anterior, se evidencia la necesidad de aprovechar subproductos de la industria alimentaria de bajo costo y con alto valor proteico; por ello el objetivo de este trabajo fue evaluar la aceptabilidad sensorial y calidad microbiológica de bebidas formuladas a base de arroz y fortificadas con proteína proveniente de plasma bovino y porcino, luego a la mejor formulación se le determinó calidad proteica. 


\section{MATERIALES Y MÉTODOS}

\section{Materias primas}

Dentro de las variedades inscritas de arroz ante la división de semillas del Instituto Colombiano Agropecuario (ICA), se encuentran CICA-4, IR-22, CICA-7, CICA-9, CICA-8, METICA-1, ORYZICA-1, ORYZICA-2, ORYZICA - 3, LLANOS-4, LLANOS-5 y LINEA-2; de la cual se ha escogió la ORYZICA-1 como variedad para la producción de la bebida debido al fácil acceso a ella en la Región Caribe. Por otro lado, la obtención de la sangre y plasma sanguíneo se obtuvo de la planta de sacrificio del municipio de Arjona-Bolívar (Colombia). Luego de la sensibilización del animal, la sangre fue recolectada directamente de la vena yugular en recipientes de vidrio de $3 \mathrm{~L}$, que contenían $12 \mathrm{~g}$ de anticoagulante (citrato de sodio), es decir, $4 \mathrm{~g}$ por cada litro de sangre recolectada. Posteriormente, los frascos con la sangre recolectada se refrigeraron a $4^{\circ} \mathrm{C}$, mediante el uso de neveras con hielo y transportados al laboratorio de Medicina de la Universidad de Cartagena, en donde la sangre se sometió a separación por centrifugación a 3000rpm durante 10min en un equipo marca, KUBOTA, modelo 1200. El plasma fue conservado en refrigeración a $4^{\circ} \mathrm{C}$.

\section{Diseño experimental}

Se desarrolló un diseño factorial $2 \times 3$ con tres repeticiones en un diseño experimental completamente al azar; cada muestra se analizó por duplicado. Los factores de estudio fueron dos: tipo de plasma adicionado y niveles de fortificación, con dos niveles para el primero (plasma de bovino y plasma de porcino) y tres para el segundo $(14,5 \%, 18,5 \%$ y $29 \%$ de plasma). Se definieron así los siguientes tratamientos: T1 = Bebida a base de arroz elaborado con $14,5 \%$ de plasma sanguíneo de bovino; T2 = Bebida a base de arroz elaborado con 18,5\% de plasma sanguíneo de bovino; T3 = Bebida a base de arroz elaborado con $29 \%$ de plasma sanguíneo de bovino; T4 = Bebida a base de arroz elaborado con 14,5\% de plasma sanguíneo de porcino; T5 = Bebida a base de arroz elaborado con $18,5 \%$ de plasma sanguíneo de porcino; T6 = Bebida a base de arroz elaborado con $29 \%$ de plasma sanguíneo de porcino y Control = Bebida a base de arroz elaborado sin plasma sanguíneo.

\section{Análisis de datos}

A los datos obtenidos en este estudio se les aplicó un análisis de varianza ANOVA empleando el programa Graph Padlnstats versión 3.1 para detectar diferencia entre medias. Las medias por tratamiento fueron comparadas utilizando las pruebas de diferencia mínima significativa. Se aceptaron diferencias a un nivel de probabilidad del $5 \%(p<0,05)$. También, se empleó el programa Minitab 16 Statistical Software para realizar gráficos de caja y gráficos de medias para intervalos HSD de Tukey.

\section{Obtención de las muestras}

La sangre de bovino y porcino se obtuvo de la planta de sacrificio del municipio de Arjona, Bolívar (Colombia); la sangre fue recolectada en recipientes de vidrio de 3 litros junto con 12 gramos de anticoagulante (citrato de sodio); es decir, 4 gramos de coagulante por cada litro de sangre recolectada. Luego de sensibilizar el animal, se recolectó la sangre directamente de la vena yugular en recipientes de vidrio. Posteriormente, los frascos con la sangre recolectada se refrigeraron a $4^{\circ} \mathrm{C}$ mediante el uso de neveras con hielo y fueron transportados al laboratorio de Medicina de la Universidad de Cartagena, en donde la sangre se sometió a separación por centrifugación a 3000rpm durante 10 minutos en un equipo marca KUBOTA, modelo 1200. El plasma fue conservado en refrigeración a $4^{\circ} \mathrm{C}$ hasta su uso en la elaboración de las bebidas (Julio et al., 2015).

\section{Elaboración de la bebida}

Siguiendo el procedimiento empleado por Camacho et al., (2014), la mezcla de arroz y agua a 85ㄷ durante 40 minutos se cocinó y luego se licuó con una trituradora mecánica de cuchillas marca Black \& Decker de 3000 rpm, se realizó un filtrado, se agregó plasma sanguíneo, se estabilizó el pH con ácido cítrico utilizando un pHmetro, luego se agregó azúcar y se estabilizó los grados Brix con un refractómetro digital, la bebida fortificada se pasterizó a $85^{\circ} \mathrm{C}$ durante 15 minutos y se envasó en caliente en recipientes plásticos, posteriormente se les aplicó un choque térmico y se almacenaron en refrigeración a $4^{\circ} \mathrm{C}$ en un refrigerador marca Haceb de $1000 \mathrm{Lt}$ por 2 días antes de realizar el panel sensorial. La formulación de los tratamientos se presenta en la Tabla 1. Los porcentajes de plasma de $14,5 \%, 18,5 \%$ y $29 \%$ se seleccionaron con el objeto de lograr que una ración de consumo de $250 \mathrm{ml}$ de la bebida supla mínimo el $10 \%, 15 \%$ y $20 \%$ respectivamente, de los requerimientos 
diarios de consumo de proteína (RDA) establecidos por la FAO para los niños de edad escolar seleccionados en este estudio (8 a 12 años) (FAO/WHO/ONU, 1991).

Tabla 1: Formulación de ingredientes utilizados para los diferentes tratamientos.

\begin{tabular}{|l|c|c|c|c|c|c|c|}
\hline \multirow{2}{*}{ Ingrediente (\%) } & \multicolumn{7}{|c|}{ Tratamientos } \\
\cline { 2 - 8 } & $\mathrm{T} 1$ & $\mathrm{~T} 2$ & $\mathrm{~T} 3$ & $\mathrm{~T} 4$ & $\mathrm{~T}$ & $\mathrm{~T}$ & Control \\
\cline { 2 - 8 } & $\begin{array}{c}14,5 \% \text { plasma } \\
\text { bovino }\end{array}$ & $\begin{array}{c}18,5 \% \\
\text { plasma } \\
\text { bovino }\end{array}$ & $\begin{array}{c}29 \% \text { de } \\
\text { plasma } \\
\text { bovino }\end{array}$ & $\begin{array}{c}14,5 \% \\
\text { plasma } \\
\text { porcino }\end{array}$ & $\begin{array}{c}18,5 \% \\
\text { plasma } \\
\text { porcino }\end{array}$ & $\begin{array}{c}29 \% \text { de } \\
\text { plasma } \\
\text { porcino }\end{array}$ & - \\
\hline Arroz & 2,5 & 2,5 & 2,5 & 2,5 & 2,5 & 2,5 & 2,5 \\
\hline Azúcar & 11 & 11 & 11 & 11 & 11 & 11 & 11 \\
\hline Agua & 72 & 68 & 57,5 & 72 & 68 & 57,5 & 86,5 \\
\hline Plasma & 14,5 & 18.5 & 29 & 14,5 & 18,5 & 29 & 0 \\
\hline
\end{tabular}

\section{Calidad microbiológica}

Se utilizó el método estándar de recuento en placa para la determinación Mesófilos aerobios (UFC/mL). Para ello se agregaron 25 gramos muestra a $225 \mathrm{~mL}$ de agua peptonada al $0,1 \% \mathrm{p} / \mathrm{v}$, y de esta forma se obtuvo la dilución $10^{-1}$. Se realizaron diluciones seriadas y se sembró en cajas de petri con medio Agar cuenta en placa, incubadas a $30 \pm 0,2^{\circ} \mathrm{C}$ por 24 horas. Posteriormente se realizaron recuentos de las colonias. Cada protocolo se realizó por duplicado (Ossa et al., 2010). Para la determinación de Coliformes Totales y Fecales (NMP/100mL) se empleó la técnica del número más probable, que consistió en la elaboración de diluciones seriadas $\left(10^{-1}, 10^{-2}\right.$ y $\left.10^{-3}\right)$. Para cada dilución se tomaron 3 tubos y se sembraron en Caldo Bilis Verde Brillante al $2 \%$. Se homogenizaron e incubaron a $43 \pm 2^{\circ} \mathrm{C}$ por 48 horas. A las 24 horas se efectuó la lectura verificando que los tubos que presentaron turbidez y presencia de gas. Las pruebas confirmativas finales para producción de gas se realizaron a las 48 horas, según lo recomendado por (Franco et al., 2013). El recuento de hongos y levaduras (UFC/mL) fue mediante el método estándar de recuento en placa. La toma de muestras para el control microbiológico se realizó a los 30 días de fabricado el producto, teniendo en cuenta parámetros microbiológicos establecidos por el Ministerio de Salud colombiano en el Decreto 7992 de 1991 para bebidas refrescantes (como la elaborada en esta investigación) con una vida útil máxima de 30 días.

\section{Evaluación de la aceptabilidad sensorial}

Para evaluar la aceptabilidad sensorial de los productos se empleó un panel de degustación no entrenado, constituido por ciento veinte (120) niños escolares de ambos sexos, en edades comprendidas entre 8 y 12 años, ya que a esta población va dirigida la bebida. Los panelistas fueron seleccionados en una escuela de la ciudad de Cartagena de Indias (Colombia). Se utilizó una escala hedónica gráfica de 5 puntos como se presenta en la Tabla 2, y se midió el grado de satisfacción que produce cada muestra al ser degustada por los panelistas, determinando así, el grado de aceptabilidad sensorial de cada formulación (Garrido et al., 2009). La prueba se realizó en dos sesiones, a cada panelista evaluador, y se le presentaron muestras en presentaciones de $20 \mathrm{~mL}$ de la bebida a base de arroz sin plasma (control) y muestras de la bebida fortificadas con plasma (formulaciones de 14,5\%; 18,5\%; 29\% con plasma bovino y $14,5 \% ; 18,5 \% ; 29 \%$ con plasma porcino).

Tabla 2: Escala hedónica para evaluar la aceptabilidad sensorial del producto.

\begin{tabular}{|c|c|c|}
\hline Escala Verbal & Escala Grafica & Puntuación \\
\hline Me gusta mucho & 5 \\
\hline $\begin{array}{c}\text { Me gusta } \\
\text { ligeramente }\end{array}$ & 4 \\
\hline $\begin{array}{c}\text { Ni me gusta ni me } \\
\text { disgusta }\end{array}$ & 3 \\
\hline $\begin{array}{c}\text { Me disgusta } \\
\text { ligeramente }\end{array}$ & \\
\hline $\begin{array}{c}\text { Me disgusta } \\
\text { mucho }\end{array}$ & \\
\hline
\end{tabular}




\section{Características fisicoquímicas y composición nutricional}

A la bebida fortificada con mayor aceptabilidad sensorial se le determinó el contenido de proteína mediante el método AOAC 955.04/90; grasa por AOAC 920.39/95; humedad por 930.15/90 y cenizas por el método AOAC 942.05/90. Adicionalmente se le evaluó la calidad nutricional. La proteína bruta se determinó empleando el método AOAC 976.05 (A.O.A.C., 2003). El índice de calidad proteica se determinó con la relación entre el requerimiento proteico en una edad determinada y el requerimiento de aminoácido más limitante de la proteína estudiada (Montero et al., 2015). Se establecieron aminoácidos esenciales por cromatografía liquida de alta resolución (HPLC), en un cromatógrafo BAS (California, USA), con detector de fluorescencia Water 474. La detección por fluorescencia se realizó utilizando una longitud de onda de excitación de 340nm y una longitud de onda de emisión de 460nm.

\section{ANÁLISIS DE RESULTADOS}

\section{Calidad microbiológica}

La Tabla 3 muestra los resultados del recuento de microorganismos de las bebidas y se comparan con la norma sanitaria que establece los criterios microbiológicos de calidad sanitaria e inocuidad para los alimentos y bebidas de consumo humano expuestos por la Comisión del Codex Alimentarius. Los resultados microbiológicos muestran que los recuentos de microorganismos para la bebida fortificada están por debajo de los máximos permitidos por el Codex Alimentarius, garantizando la inocuidad del producto, lograda probablemente con el ajuste de $\mathrm{pH}$ y tratamiento térmico. Los resultados coinciden con los obtenidos por Benítez et al., (2011) para una galleta elaborada con plasma sanguíneo de bovino.

Tabla 3: Recuento de microorganismos para las bebidas fortificadas

\begin{tabular}{|c|c|c|c|c|c|c|c|}
\hline Microorganismos & \multicolumn{3}{|c|}{$\begin{array}{c}\text { Bebida fortificada con } \\
\text { plasma bovino (\%) }\end{array}$} & \multicolumn{3}{c|}{$\begin{array}{c}\text { Bebida fortificada con } \\
\text { plasma porcino (\%) }\end{array}$} & $\begin{array}{c}\text { Codex } \\
\text { Alimentarius }\end{array}$ \\
\cline { 2 - 7 } & 14 & 18,5 & 29 & 14 & 18,5 & 29 & $<3$ \\
\hline Recuento de Mesófilos aerobios (UFC/mL) & $<3$ & $<5$ & $<3$ & $<10$ & $<3$ & $<3$ \\
\hline Recuento de Mohos (UFC/mL) & $<1$ & $<1$ & $<4$ & $<2$ & $<1$ & $<1$ & 8 \\
\hline Recuento Coliformes totales (NMP/100mL) & $<1$ & $<1$ & $<1$ & $<1$ & $<1$ & $<1$ & 2 \\
\hline
\end{tabular}

\section{Evaluación de la aceptabilidad sensorial}

En la Tabla 4 se presenta la media de la prueba de aceptabilidad sensorial de cada una de las bebidas. Los resultados muestran que el nivel de fortificación no afecta significativamente la aceptabilidad sensorial de la bebida refrescante a base de arroz fortificada con plasma bovino. Sin embargo si hay significancia estadística $(p<0,05)$ en el efecto que produce el tipo de plasma en la aceptabilidad sensorial del producto. La bebida sin fortificar presentó la media más elevada; además, no hubo diferencia estadística significativa $(p<0,05)$ en la aceptación de la bebida sin fortificar y la bebida fortificada con $29 \%$ de plasma bovino (T3). Para conocer la variabilidad de los datos se realizaron gráficas de caja y bigotes para las respuestas dadas por los panelistas para las bebidas refrescantes a base de arroz fortificadas con plasma de bovino (Figura 1) y las bebidas refrescantes a base de arroz fortificadas con plasma de porcino (Figura 2).

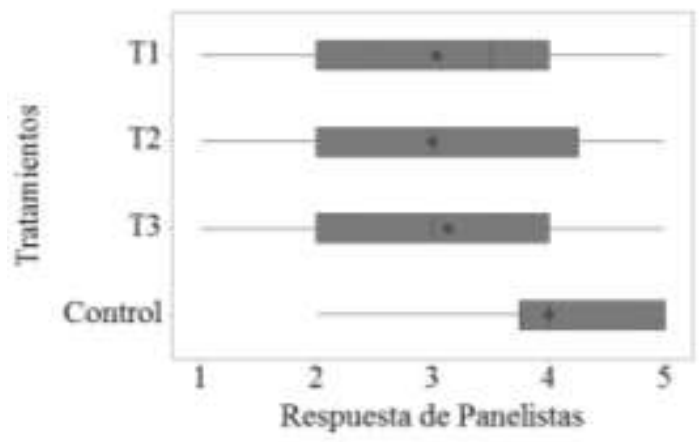

Fig. 1: Variabilidad de las calificaciones asignadas por los panelistas a la bebida refrescante a base de arroz fortificada con plasma bovino.

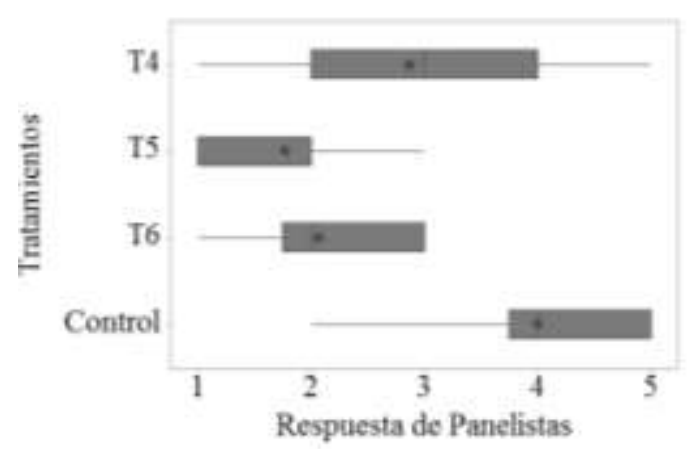

Fig. 2: Variabilidad de las calificaciones asignadas por los panelistas a la bebida refrescante a base de arroz fortificada con plasma porcino. 
Tabla 4: Aceptabilidad sensorial debido a los diferentes niveles de fortificación y proteína plasmática $(n=120)$

\begin{tabular}{|c|c|c|c|c|}
\hline Plasma & $14,5 \%$ plasma & $18,5 \%$ plasma & $29 \%$ de plasma & Bebida de arroz sin fortificar \\
\hline \multirow{2}{*}{ Bovino } & $\mathrm{T} 1$ & $\mathrm{~T} 2$ & $\mathrm{~T}$ & Control \\
\cline { 2 - 4 } & $3,03 \pm 1,4^{\mathrm{a}}$ & $3,00 \pm 1,4^{\mathrm{a}}$ & $3,13 \pm 1,3^{\mathrm{a}^{\mathrm{d}}}$ & \multirow{2}{*}{$4 \pm 1,0^{\mathrm{d}}$} \\
\hline \multirow{2}{*}{ Porcino } & $\mathrm{T} 4$ & $\mathrm{~T} 5$ & $\mathrm{~T} 6$ & \\
\cline { 2 - 4 } & $2,86 \pm 1,2^{\mathrm{a}^{\mathrm{c}}}$ & $1,76 \pm 0,8^{\mathrm{b}}$ & $2,06 \pm 0,7^{\mathrm{bc}}$ & \\
\hline
\end{tabular}

La Figura 1 nos indica que los tratamientos T1 y T3 presentan similitud en la variabilidad de sus datos, ya que las cajas son de tamaño muy similar. El tratamiento T2 fue el que más variabilidad mostró en las respuestas dadas por los panelistas, ya que la caja que lo representa es la de mayor tamaño. Por el contrario, el control tiene la menor variabilidad de aceptación, ya que la caja que lo representanta es la más pequeña de todas. La respuesta de los panelistas en general fue muy variada debido a que sus puntuaciones fueron de 1 a 5 en la mayoría de los tratamientos. En contraste, la Figura 2 muestra que los tratamientos T5 y T6 de plasma porcino mostraron menor variabilidad. Además, se observa que a la mayor parte de los panelistas les disgustó la bebida fortificada con $18 \%$ de plasma porcino (T5), y a ningún panelista le agradó el tratamiento fortificado con $29 \%$ de plasma porcino (T6) pues este tratamiento no obtuvo calificaciones de 4 o 5 . También se observa que el tratamiento con $14,5 \%$ de plasma porcino (T4), fue el que más variabilidad de aceptación presentó, ya que los panelistas le asignaron desde la puntuación más baja (1) a la más alta (5).

Para conocer cuál de los tratamientos fue el mejor calificado se emplearon gráficos de medias para intervalos HSD de Tukey para las bebidas fortificadas con plasma bovino y porcino (Figura 3 y 4 respectivamente). De acuerdo a la Figura 3, se observa que a pesar de que las medias de estos tratamientos están cercanas a solaparse, el tratamiento con $29 \%$ de plasma bovino (T3) fue el que tendió a mostrar una media levemente más alta, aunque no hubo diferencia estadística significativa $(p<0,05)$ en la aceptabilidad sensorial de las diferentes bebidas fortificadas con plasma bovino por parte de los panelistas. En la Figura 4, se observa que ninguna de las muestras fortificadas con plasma porcino tuvo aceptabilidad sensorial, pues todas las medias están por debajo de 3. Los tratamientos T5 y T6 poseen las medias más bajas, lo que significa que fueron las que más disgustaron a los panelistas. El tratamiento que menos disgustó fue la bebida fortificada con 14,5\% (T4) que poseía la media más alta. Por su parte el control fue el tratamiento mejor calificado, con una media de 2,06 $\pm 0,7$. Una posible estrategia para mejorar las características organolépticas de las bebidas adicionadas con plasma en general, es el empleo de enmascarantes de sabor tal como lo han implementado otros autores con el empleo de saborizantes de chocolate, fresa, entre otros (Barboza et al., 1994; 2005; Camacho et al., 2014Julio et al., 2015; Montero et al., 2015).

En estudios similares Benítez et al., (2011) reportaron una aceptabilidad sensorial superior al 79\% para una galleta formulada con harina de yuca y plasma sanguíneo de bovino. Por su parte Márquez et al., (2006) también reportaron una alta aceptabilidad sensorial para un embutido con agregado de piel de pollo emulsificado con sangre de bovino. Del Rio et al., (1980) reportaron que el plasma sanguíneo de ave afectó negativamente algunas características sensoriales del pan elaborado con adición de plasma. Considerando como patrón de aceptabilidad sensorial una puntación igual o superior de tres (Benítez et al., 2011), la bebida fortificada con $29 \%$ de plasma bovino (T3) fue aceptada por el $66,67 \%$ de los panelistas, siendo la bebida fortificada con plasma animal mayor aceptada. El control obtuvo el mayor porcentaje de aceptación con un $90 \%$ (Ver Figura 5).

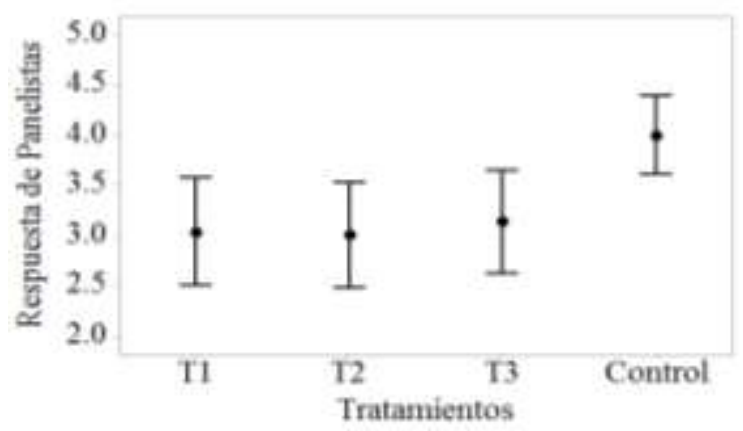

Fig. 3: Medias para intervalos HSD de Tukey para bebida refrescante a base de arroz fortificada con plasma bovino.

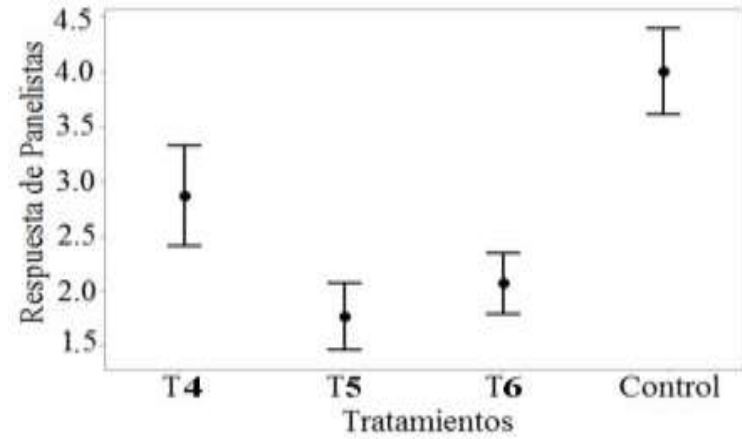

Fig. 4: Medias para intervalos HSD de Tukey para bebida refrescante a base de arroz fortificada con plasma porcino. 


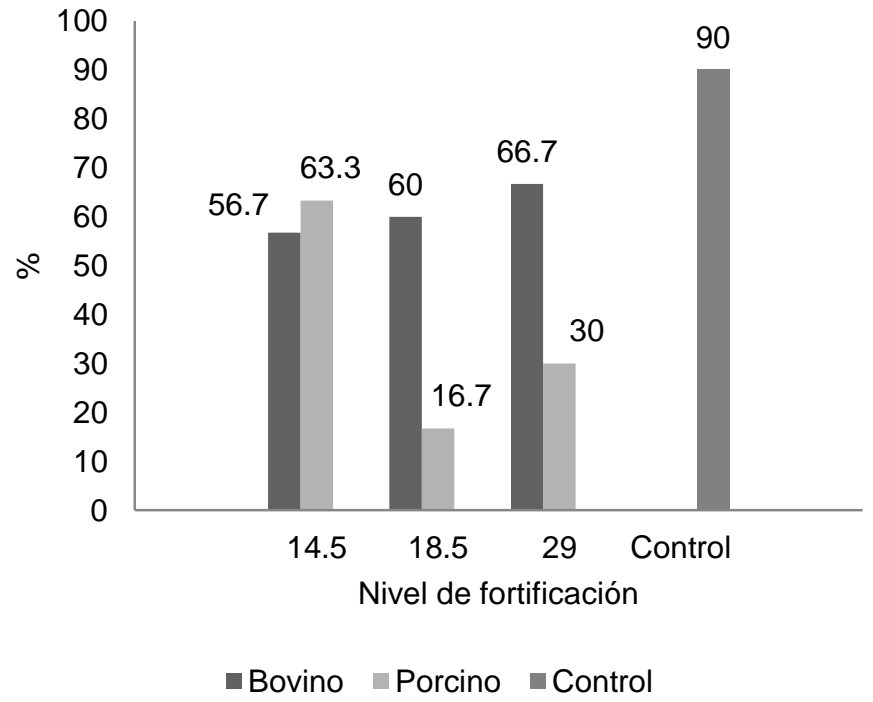

Fig. 5: Porcentaje de aceptación de la bebida

\section{Características fisicoquímicas y composición nutricional}

La Tabla 5 muestra las características fisicoquímicas y composición nutricional del mejor tratamiento. A la bebida fortificada con $29 \%$ de plasma bovino (T3) por ser la bebida fortificada de mayor aceptación se le evaluó

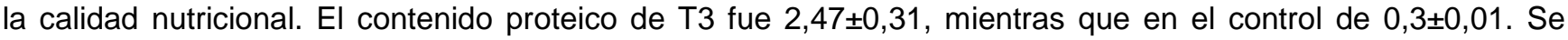
evidenció que en T3 el contenido de proteína fue significativamente mayor $(p<0,05)$ que el control. El aumento en el contenido proteico de la bebida de arroz cuando se fortificó con plasma coincide con los resultados reportados por otros autores (Barboza et al., 2005; Camacho et al., 2014; Montero et al., 2015), quienes encontraron que al incorporar $40 \%$ de proteína plasmática en un producto formulado con maíz tierno el contenido proteico incrementó de $3,54 \%$ en el producto formulado sin plasma bovino a $6,47 \%$ en el producto que contenía plasma.

Tabla 5: Características fisicoquímicas y composición nutricional del mejor tratamiento

\begin{tabular}{|c|c|}
\hline Características & Valor promedio \\
\hline Proteína & $2,47 \pm 0,31$ \\
\hline Grasa & $0,45 \pm 0,01$ \\
\hline Humedad & $90,95 \pm 1,43$ \\
\hline Cenizas & $1,15 \pm 0,18$ \\
\hline
\end{tabular}

\section{Aminoácidos esenciales}

La media de la determinación de aminoácidos esenciales contenidos en las proteínas de la bebida fortificada con plasma bovino se presentan en la Tabla 6; y además, se comparan con los perfiles de aminoácidos del grano de arroz (Romo et al., 2006), plasma de diferentes especies (Bracho et al., 2001) y el modelo de referencia basado sobre las necesidades de aminoácidos indispensables para los escolares de 6 a 12 años, rango en el cual caen la población de niños que participaron en este estudio (escolares de 8 a 12 años) (FAO/WHO/ONU, 1991).

Las proteínas de arroz y plasma sanguíneo de este estudio, al ser incompletas por si solas, pueden ser combinadas con el objetivo que compensen entre si las deficiencias que presentan por separado en cuanto a aminoácidos esenciales; ya que de esta manera se puede crear una fuente proteica que suministre estos al ser humano (Williams, 1995). Teniendo en cuenta lo anterior, y de acuerdo a la Tabla 6, la fortificación de la bebida refrescante a base de arroz con plasma de bovino y porcino, permitió crear un producto que si cumple con un adecuado suministro de aminoácidos esenciales, lo cual evidencia el carácter sinérgico de la combinación de las materias primas de este estudio. Cabe resaltar que el valor nutritivo de la bebida refrescante fortificada con 
plasma bovino fue mayor en el presente estudio, y además mostró mejor balance de aminoácidos que en el arroz. Estos resultados coinciden con los reportados por Barboza et al., (2005), quienes en su estudio de evolución del efecto de la incorporación de proteína plasmática sobre la calidad proteica de un producto con maíz, notaron que al combinar el maíz con el plasma, se llegaba a aumentar la calidad del producto final, y además se lograba un equilibrio adecuado de aminoácidos, obteniendo un alimento con un mayor valor nutritivo, donde el plasma le aporto lisina al maíz y el maíz aporto metionina al plasma, complementándose entre sí, al igual que en esta investigación.

Tabla 6: Comparación de valores promedios de aminoácidos esenciales del arroz, plasma y la bebida de arroz fortificada con plasma sanguíneo de bovino y porcino en gramos de aminoácido/100 gramos de proteína

\begin{tabular}{|l|c|c|c|c|}
\hline Aminoácido & $\begin{array}{c}\text { Grano } \\
\text { de arroz }\end{array}$ & $\begin{array}{c}\text { Plasma } \\
\text { bovino }\end{array}$ & $\begin{array}{c}\text { Bebida fortificada con } \\
\text { plasma bovino (T3) }\end{array}$ & $\begin{array}{c}\text { Requerimientos de aminoácidos } \\
\text { para niños edad escolar } \\
\text { FAO/WHO/ONU (1991) }\end{array}$ \\
\hline Isoleucina & 4,1 & 2,56 & $2,9^{\mathrm{a}}$ & 2,8 \\
\hline Leucina & 8,2 & 5,96 & $6,2^{\mathrm{a}}$ & 4,4 \\
\hline Lisina & 3,8 & 7,18 & $7,1^{\mathrm{a}}$ & 4,4 \\
\hline Metionina+cisteína & 3,6 & 0,21 & $3,4^{\mathrm{a}}$ & 2,2 \\
\hline Fenilalanina+Tirosina & 9,1 & 6,11 & $6,7^{\mathrm{b}}$ & 2,2 \\
\hline Treonina & 4,1 & 5,34 & $5,5^{\mathrm{a}}$ & 2,8 \\
\hline Valina & 5,8 & 3,85 & $4,3^{\mathrm{a}}$ & 2,5 \\
\hline Histidina & 2,6 & 5,12 & $5,2^{\mathrm{a}}$ & 1,9 \\
\hline
\end{tabular}

La calificación de una proteína como nutricionalmente adecuada depende principalmente de su capacidad para satisfacer los requerimientos de nitrógeno y de aminoácidos esenciales. Sobre la base de estas consideraciones, se puede demostrar que cuando las proteínas son comparadas con los patrones de requerimientos de aminoácidos esenciales para cada edad, una proteína puede resultar inadecuada para el niño y ser adecuada para el adulto (Romo et al., 2006).

El valor biológico nutritivo de una proteína, para una población de determinada edad, depende de su composición en aminoácidos esenciales (Romo et al., 2006). En ese orden de ideas fue necesario determinar el aminoácido de la proteína de la bebida de arroz fortificada con plasma de bovino que resulta ser más deficitario de acuerdo a los requerimientos de los niños de edad escolar que fue la población objeto de este estudio. En la Tabla 7 se presentan los aminoácidos esenciales de las proteínas de la bebida de arroz fortificada que resulta limitante para niños de edad escolar de 6 a 12 años y se comparan con los datos para el grano de arroz (Romo et al., 2006) y los requerimientos de aminoácidos para niños edad escolar (FAO/WHO/ONU, 1991).

Tabla 7: Índice de calidad proteica de la bebida a base de arroz fortificada con plasma bovino con relación a los requerimientos de aminoácidos y proteína para de niños en edad escolar (6-12 años).

\begin{tabular}{|l|c|c|c|}
\hline Aminoácidos esenciales & $\begin{array}{c}\text { Grano de } \\
\text { arroz }\end{array}$ & $\begin{array}{c}\text { Bebida fortificada con } \\
\text { plasma bovino }\end{array}$ & $\begin{array}{c}\text { Requerimientos de aminoácidos para niños } \\
\text { edad escolar FAO/WHO/ONU (1991) }\end{array}$ \\
\hline Isoleucina & 0,7 & 0,96 & 2,8 \\
\hline Leucina & 0,5 & 0,70 & 4,4 \\
\hline Lisina & 1,2 & 0,61 & 4,4 \\
\hline Metionina+ cisteína & 0,6 & 0,64 & 2,2 \\
\hline Fenilalanina+Tirosina & 0,2 & 0,32 & 2,2 \\
\hline Treonina & 0,7 & 0,50 & 2,8 \\
\hline Valina & 0,4 & 0,58 & 2,5 \\
\hline Histidina & 0,7 & 0,36 & 1,9 \\
\hline Índice de calidad & 82,5 & 100 & - \\
\hline
\end{tabular}

Según la FAO se considera 0,99g/kg para los niños de 6 a 12 años la dosis inocua de proteínas de referencia. Los resultados muestran que las proteínas de la bebida enriquecida con el plasma porcino son deficientes en isoleucina y leucina para el requerimiento de niños de edad escolar. La bebida fortificada con plasma bovino satisface todos los requerimientos de aminoácidos esenciales de los niños de edad escolar, por lo cual se considera que la proteína tiene una calidad de $100 \%$ para este grupo etario. 


\section{CONCLUSIONES}

El tipo de plasma adicionado y los niveles de fortificación afectan significativamente la aceptabilidad sensorial de las bebidas refrescantes a base de arroz. La bebida fortificada con $29 \%$ de plasma bovino fue el tratamiento con mayor aceptabilidad sensorial por parte de los panelistas. Es posible la fortificación de bebidas con concentraciones de $29 \%$ de plasma bovino, pero se requiere adicionar concentraciones y tipos de saborizantes que permitan mejorar su sabor y aceptabilidad sensorial. El perfil de aminoácidos de la fortificación de la bebida a base de arroz no se ven afectados en comparación con la bebida sin fortificar. La bebida fortificada con $29 \%$ de plasma bovino posee el mayor aporte proteico y satisface los requerimientos de aminoácidos esenciales de los niños de edad escolar. La fortificación de la bebida refrescante a base de arroz con plasma de bovino permitió crear un producto que si cumple con un adecuado suministro de aminoácidos esenciales, lo cual evidencia el carácter sinérgico de la combinación de las materias primas de este estudio. Las bebidas cumplen con los estándares microbiológicos. La importancia de este trabajo radica en que se implementó el uso de un plasma sanguíneo animal diferente (cerdo) al utilizado convencionalmente por otros autores (bovino). Además se demostró que es posible incorporar porcentajes de plasma hasta del $29 \%$, que permitan suplir hasta el $20 \%$ de los requerimientos diarios de consumo de proteína (RDA) establecidos por la FAO para los niños de edad escolar seleccionados en este estudio (8 a 12 años). Se espera que con los resultados de esta investigación se puedan presentar alternativas a los programas gubernamentales destinado a niños con desnutrición proteica.

\section{AGRADECIMIENTOS}

Los autores agradecen a COLCIENCIAS por la financiación del proyecto "Desarrollo de productos de pastelería empleando como base pasta de ajonjolí (Sésamum indicum) y plasma sanguíneo de bovino como surfactante" en la Convocatoria 664 "CONVOCTORIA PARA EL APOYO A LA INNOVACIÓN EN GASTRONOMÍA - 2014" de donde se derivan los resultados de este artículo.

\section{REFERENCIAS}

A.O.A.C., Métodos de análisis de la asociación oficial de química analítica para determinar humedad, fibra, cenizas, grasa y proteína., Washington, U.S.A., Chapter 32: 1, 2, 5 y 14 (2003)

Barboza, Y. y otros cinco autores, Efecto de la incorporación de proteína plasmática sobre la composición química y calidad proteica de un producto formulado con maíz tierno, Rev. Cient. Fac. Ciencias Vet. LUZ, 15(6), 536-542 (2005)

Barboza, Y. y otros cuatro autores, Utilización del plasma sanguíneo de bovino como fuente proteica en la formulación de un medio de cultivo pata lactobacilos, Revista Científica Facultad de Ciencias Veterinarias 4: 5559 (1994)

Benítez, B. y otros cinco autores, Calidad nutricional y aceptabilidad de un producto formulado con carne de pollo deshuesada mecánicamente, plasma y glóbulos rojos de bovino, ALAN, 52(3), 307-312 (2002)

Benítez, B. y otros seis autores, Calidad microbiológica de una galleta formulada a base de harina de yuca y plasma de bovino, Rev.Fac. Agron. LUZ, 28(2), 260-272 (2011)

Bracho, M., E. Márquez y B. Arias, Estudio comparativos del contenido de aminoácidos esenciales en sangre de bovino y cerdo, Rev. Cient. Fac. Ciencias Vet. LUZ, 11(2), 133-138 (2001)

Camacho, A, P.M. Montero y M. Duran, Efecto de la adición de plasma sanguíneo de bovino en el contenido protéico, aceptabilidad y calidad microbiológica de una bebida a base de arroz, Revista Alimentos Hoy, 22(31), 104-108 (2014)

Del Rio, M. y otros cuatro autores, Chicken blood plasma proteins: physicochemical, nutritional and functional properties, Journal of Food Science 45(1), 17-20 (1980)

FAO/OMS/UNU, Necesidades de energía y de proteínas, Serie Inf. Técn. № 724, 220 p. Reunión Consultiva Conjunta FAO/OMS/UNU de Expertos en Necesidades de Energía y de Proteínas, Roma, Italy (1991) 
Figueroa, O., J. Zapata y G. Gutiérrez, Modelamiento de la cinética de hidrólisis enzimática de proteínas del plasma bovino, Revista EIA, 9(17), 71-84 (2012)

Franco, P. y otros tres autores, Determinación de Escherichia coli e identificación del serotipo O157:H7 en carne de cerdo comercializada en los principales supermercados de la ciudad de Cartagena, Rev. Lasallista de Investigación, 10(1), 91-100 (2013)

Garrido, F. y otros cinco autores, Aceptabilidad de sopas deshidratadas de leguminosas adicionadas de realzadores del sabor (umami), Revista Chilena de Nutrición, 36(4), 1105-1112 (2009)

Gómez, L., O. Figueroa y J. Zapata, Actividad antioxidante de hidrolizados enzimáticos de plasma bovino obtenidos por efecto de Alcalasa® 2.4 L, Información Tecnológica, 24(1), 33-42 (2013)

González, D., D. Castro y D. Castillo, Valor nutricional, importancia para la salud y estabilidad del arroz integral, Ciencia y Tecnología de Alimentos 17(3), 73-80 (2007)

Hurtado, S. y otros seis autores, Use of porcine blood plasma in "phosphate-free frankfurters", Procedia Food Science, 1, 477-482 (2011)

Isaza, J. y otros cuatro autores, Producción y propiedades funcionales de plasma bovino hidratado en embutido tipo salchichón, Revista Colombiana de Ciencias Pecuarias, 23(2), 199-206 (2010)

Julio, L.C., P.M. Montero y D. Acevedo, Calidad y aceptabilidad de chorizos formulados con plasma sanguíneo bovino y pasta de ajonjolí. Información Tecnológica, 26(3), 25-32 (2015)

Márquez, E. y otros cinco autores, Formulación de un embutido con agregado de piel de pollo emulsificada con sangre de bovino, Revista Científica FCV-LUZ, 16(4), 438-444 (2006)

Montero, P.M., Y.A. Marrugo y L.C. Julio, Protein Quality of Rice Drinks Fortified with Bovine and Porcine Blood Plasma, Revista Facultad Nacional de Agronomía, 68(1), 7487-7496 (2015)

Ossa, J., A. Coral y M. Vanegas, Microbiota de jamones de cerdo cocidos asociada al deterioro por abombamiento del empaque, Rev. MVZ Córdoba, 15(2), 2078-2086 (2010)

Parés, D., E. Saguer y C. Carretero, 9 - Blood by-products as ingredients in processed meat. Processed Meats, Improving Safety, Nutrition and Quality A volume in Woodhead Publishing Series in Food Science, Technology and Nutrition, 218-242 (2011)

Rangel, L., y otros cuatro autores, Utilización de tripolifosfato como anticoagulante y su efecto sobre las propiedades emulsificantes del plasma, Rev. Cient. Fac. Ciencias Vet. LUZ, 5(2), 111-116 (1995)

Rodríguez, L., A. Pérez y M. Campderrós, Plasma bovino tecnológicamente mejorado para aplicaciones alimenticias, La Alimentación Latinoamericana, 93, 44-48 (2011)

Romo, S. y otros tres autores, Potencial nutricional de harinas de quinua (Chenopodium quinoa W.) variedad piartal en los andes colombianos primera parte, Revista de Biotecnología en el Sector Agrario y Agroindustrial, $4(1), 112-125(2006)$

Williams. S.R., Basic Nutrition and Diet Therapy, 10a Edición, 720 pp. Mosby Publishers, St. Louis, USA (1995) 